\title{
Prognostic Factor and Survival Benefit of Adjuvant Chemotherapy in Stage IIA Colon Cancer
}

\author{
Mok-Won Lee, Jin-Soo Kim, Ji-Yeon Kim, Kyung-Ha Lee \\ Department of Surgery, Chungnam National University Hospital, Daejeon, Korea
}

Purpose: There is no clear evidence of the benefit of adjuvant chemotherapy (AC) in stage IIA colon cancer. Therefore, we aimed to evaluate the prognostic factors and survival benefit of $\mathrm{AC}$ in this disease.

Methods: A retrospective data collection for patients who underwent radical surgery for colon cancer between January 2008 and December 2015 was undertaken. The cohort was divided into the no-AC and AC groups.

Results: We included 227 patients with stage IIA colon cancer in our study cohort, including 67 and 160 patients in the no-AC and AC groups, respectively. The number of retrieved lymph nodes and the presence of tumor complications as obstruction or perforation were independent risk factors for survival. In the no-AC group, there was a significant difference in survival according to the number of retrieved lymph nodes. In the AC group, there were significant differences in survival according to sidedness and preoperative carcinoembryonic antigen (CEA). There was no significant difference in survival between the no-AC and the AC groups.

Conclusion: The number of retrieved lymph nodes and the presence of tumor complications were prognostic factors for stage IIA colon cancer but lymphovascular and perineural invasion were not. Sidedness and preoperative CEA could be used as factors to predict the benefit of adjuvant chemotherapy. Currently, it is believed that there is no benefit of AC for stage IIA colon cancer. Further studies are needed to determine the survival benefit of adjuvant chemotherapy in stage IIA colon cancer.

Keywords: Colonic neoplasms; Neoplasm staging; Adjuvant chemotherapy; Survival; Prognosis

\section{INTRODUCTION}

Colorectal cancer is the second most common cancer in Korea $(28,111 / 100,000,12.1 \%)$ after stomach cancer $(29,685 / 100,000$, $12.8 \%$ ) [1]. Localized disease, which is defined as tumor limited to the colon or rectum without any spread to adjacent organs or lymph node (LN) according to the summary stage by SEER (surveillance, epidemiology, and end results) program, corresponding to stage I or IIA based on the American Joint Committee on Cancer (AJCC) 8th edition accounts for $36.1 \%$ of all colorectal can-

Received: May 16, 2020 - Revised: Aug 20, 2020 - Accepted: Sep 3, 2020 Correspondence to: Kyung-Ha Lee, M.D.

Department of Surgery, Chungnam National University Hospital, 282 Munwha-ro, Jung-gu, Daejeon 35015, Korea

Tel: +82-42-280-7175, Fax: +82-42-257-8024

E-mail: IIIIlkh@cnuh.co.kr

ORCID: https://orcid.org/0000-0002-0035-9000

(C) 2021 The Korean Society of Coloproctology

This is an open-access article distributed under the terms of the Creative Commons Attribution NonCommercial License (https://creativecommons.org/licenses/by-nc/4.0) which permits unrestricted noncommercial use, distribution, and reproduction in any medium, provided the original work is properly cited. cers, and its 5-year overall survival rate was 94.5\% [2].

Worldwide treatment guidelines currently recommend adjuvant chemotherapy (AC) with capecitabine of 5-fluorouracil (FU)/leucovorin with or without oxaliplatin for pathologic T3N0M0 (stage IIA) colon cancer with high risk for systemic recurrence. However, due to lack of clear evidence, there is a controversy regarding its benefit over its adverse effects. Apart from the generally known adverse effects of cytotoxic agents which include stomatitis, nausea, vomiting, or diarrhea, accumulation of oxaliplatin can cause long-lasting or permanent peripheral neuropathy and severely interfere with the quality of life. Despite the favorable survival outcomes of stage IIA colon cancer, a significant number of patients may not get any benefits; rather, they can be harmed by overtreatment. Moreover, this results in personal and national economical wastage due to unnecessary treatment. Recently, the International Duration Evaluation of Adjuvant Chemotherapy (IDEA) collaboration group has reported the oncologic non-inferiority of 3-month to 6-month CAPOX (capecitabine and oxaliplatin) regimen with significantly decreased neurotoxicity in stage III [3] and high-risk stage II colon cancer patients [4], and has formulated 
major guidelines which many physicians have incorporated into their practice. However, there is a lack of research about the specific subgroup of stage IIA colon cancers that can be oncologically safe without any AC.

In the last few decades, universal standardization of complete mesocolic excision and central vessel ligation have improved the surgical outcome and the accuracy of pathologic staging [5]. Besides, the development of tests including computed tomography, magnetic resonance imaging, or positron emission tomography for preoperative clinical staging has increased the rate of diagnosis of synchronous metastasis at the initial staging. This has led to a decrease in the misdiagnosis of advanced colon cancers as T3NOM0 stage IIA colon cancer. Consequently, it can be assumed that the prognosis of stage IIA colon cancers today can be more favorable than in the past. Therefore, the prognosis and prognostic factors of stage IIA colon cancer need to be reevaluated, and the current indication for AC based on the research of the past needs to be revalidated.

\section{METHODS}

\section{Patients and treatment}

Retrospective data collection was done using electronic medical records for all patients that underwent radical surgery for colon cancer between January 2008 and December 2015. The cohort was achieved based on the following inclusion criteria; histologically confirmed adenocarcinoma of colon (above $15 \mathrm{~cm}$ from anal verge), successful R0 resection with adequate LN dissection, and pathologic stage IIA (pT3N0M0) according to AJCC 8th edition. The exclusion criteria were as follows; distant metastasis, preoperative chemotherapy or radiotherapy, familial adenomatous polyposis or hereditary nonpolyposis colorectal cancer, and diagnosis of other malignancies which can affect survival. Patients were recommended AC if they had 1 or more risk factors for recurrence including poorly differentiated histology, lymphatic/vascular invasion, perineural invasion, less than 12 LNs examined, perforation or obstruction according to National Comprehensive Cancer Network (NCCN) guideline. AC was omitted if a patient was contraindicated for chemotherapy or refused chemotherapy for any reason including medical or surgical problems. This study was approved by the Institutional Review Board of Chungnam National University Hospital (No. 2020-02-040), and the requirement for documentation of written informed consent was waived.

\section{Subgroups}

The cohort was divided into a no-AC group and an AC group. Chemotherapeutic regimens included 1 year of doxifluridine $(2,250 \mathrm{mg} /$ day), 5 cycles of tegafur/uracil (tegafur $300 \mathrm{mg}$, uracil $672 \mathrm{mg}$ per day), 8 cycles of capecitabine $\left(2,500 \mathrm{mg} / \mathrm{m}^{2} /\right.$ day $), 12$ cycles of modified FOLFOX-6 (oxaliplatin $85 \mathrm{mg} / \mathrm{m}^{2}$, leucovorin $400 \mathrm{mg} / \mathrm{m}^{2}, 5$-FU $400 \mathrm{mg} / \mathrm{m}^{2}$ bolus, 5 -FU $2,400 \mathrm{mg} / \mathrm{m}^{2} /$ day over $46-48$ hours), or 12 cycles of modified FOLFOX-7 (oxaliplatin 85 $\mathrm{mg} / \mathrm{m}^{2}$, leucovorin $400 \mathrm{mg} / \mathrm{m}^{2}, 5-\mathrm{FU} 2,400 \mathrm{mg} / \mathrm{m}^{2} /$ day over $46-$ 48 hours). The patients who had completed less than half of the aforementioned schedule were included in the no-AC group.

\section{Statistical analysis}

The clinicopathologic factors of the 2 groups were compared by the chi-square test. Multivariate analyses of traditional risk factors were conducted by Cox regression analysis. The correlation between the clinicopathologic factors and survival outcomes (5-year disease-free survival [5Y-DFS] and 5-year overall survival [5YOS]) were analyzed separately in each group by Kaplan-Meier survival analysis and log-rank test. All statistical analyses were performed using IBM SPSS Statistics ver. 20 (IBM Corp., Armonk, NY, USA).

\section{RESULTS}

The total number of the stage IIA colon cancer patients in the cohort was 227. The number of patients in the no-AC group and AC group were 67 and 160 (67 patients had oral 5-FU, 40 patients had capecitabine, and 23 patients had FOLFOX), respectively. Median follow-up period was 66.74 months (0.69-142.19 months).

The comparison of clinicopathologic factors between both groups is presented in Table 1. The rate of male patients, young patients (less than 75 years old), and patients with improved performance (American Society of Anesthesiologists physical status classification I or II) were significantly higher in the AC group than in the no- $\mathrm{AC}$ group $(\mathrm{P}=0.045, \mathrm{P}<0.001$, and $\mathrm{P}=0.010$, respectively). There was no significant difference in the presence of tumor complications (obstruction or perforation), carcinoembryonic antigen (CEA) level, tumor size, number of retrieved LNs, tumor differentiation, lymphovascular invasion, or perineural invasion between the groups.

The multivariate analyses of traditional risk factors are presented in Table 2. The number of retrieved LNs was the independent risk factor for both 5Y-DFS and 5Y-OS, and the presence of tumor complications was the independent risk factor for 5Y-OS.

The correlation between clinicopathologic factors and survival outcomes of the no-AC group are presented in Table 3. Patients with less than 12 retrieved LNs had a significantly poorer $5 Y-D F S$ (78.6\% vs. $100 \%, \mathrm{P}=0.001)$ and $5 \mathrm{Y}-\mathrm{OS}(78.6 \%$ vs. $100 \%$, $\mathrm{P}=0.001)$ than the patients with 12 or more retrieved LNs. There was no significant difference in 5Y-DFS and 5Y-OS according to tumor sidedness, the presence of tumor complications, CEA level, tumor size, tumor differentiation, lymphovascular invasion, or perineural invasion.

The correlation between clinicopathologic factors and survivals of the AC group is presented in Table 4. Patients with right colon cancer (from cecum to splenic flexure) had significantly better 5Y-DFS $(97.8 \%$ vs. $85.6 \%, \mathrm{P}=0.024)$ and $5 \mathrm{Y}-\mathrm{OS}(100 \%$ vs. $83.2 \%$, $\mathrm{P}=0.019$ ) than patients with left colon cancer (from descending 
Table 1. Comparison of clinicopathologic factors between the no-AC group and AC group

\begin{tabular}{|c|c|c|c|}
\hline Variable & No-AC $(n=67)$ & $A C(n=160)$ & P-value \\
\hline Sex & & & 0.045 \\
\hline Male & $33(49.3)$ & $100(62.5)$ & \\
\hline Female & $34(50.7)$ & $60(37.5)$ & \\
\hline Age (yr) & & & $<0.001$ \\
\hline$<75$ & $42(62.7)$ & $136(85.0)$ & \\
\hline$\geq 75$ & 25 (37.3) & $24(15.0)$ & \\
\hline ASA PS classification & & & 0.010 \\
\hline I, II & $51(76.1)$ & $143(89.4)$ & \\
\hline$\| I I-V$ & $16(23.9)$ & $17(10.6)$ & \\
\hline Sidedness & & & 0.197 \\
\hline Right & $26(38.8)$ & $51(31.9)$ & \\
\hline Left & 41 (61.2) & $109(68.1)$ & \\
\hline Tumor complication & & & 0.399 \\
\hline No & $59(88.1)$ & $137(85.6)$ & \\
\hline Yes & $8(11.9)$ & $23(14.4)$ & \\
\hline CEA level (ng/mL) & & & 0.564 \\
\hline$<5$ & $43(72.9)$ & $104(72.7)$ & \\
\hline$\geq 5$ & $16(27.1)$ & 39 (27.3) & \\
\hline Tumor size (cm) & & & 0.337 \\
\hline$<5$ & $35(52.2)$ & $77(48.1)$ & \\
\hline$\geq 5$ & $32(47.8)$ & $83(51.9)$ & \\
\hline No. of retrieved LN & & & 0.489 \\
\hline$<12$ & $15(22.4)$ & $34(21.3)$ & \\
\hline$\geq 12$ & $52(77.6)$ & $126(78.8)$ & \\
\hline Differentiation & & & 0.182 \\
\hline Well, moderate & $63(94.0)$ & $156(97.5)$ & \\
\hline Poor, mucinous & $4(6.0)$ & $4(2.5)$ & \\
\hline Lymphovascular invasion & & & 0.245 \\
\hline No & $3(4.5)$ & $3(1.9)$ & \\
\hline Yes & $64(95.5)$ & $157(98.1)$ & \\
\hline Perineural invasion & & & 0.331 \\
\hline No & $24(35.8)$ & $64(40.0)$ & \\
\hline Yes & 43 (64.2) & $96(60.0)$ & \\
\hline
\end{tabular}

Values are presented as number (\%).

AC, adjuvant chemotherapy; ASA, American Society of Anesthesiologists; PS, physical status; CEA, carcinoembryonic antigen; LN, lymph node.

to sigmoid colon). Moreover, patients with normal CEA ( $<5 \mathrm{ng} /$ $\mathrm{mL}$ ) had significantly better $5 \mathrm{Y}-\mathrm{DFS}(94.9 \%$ vs. $72.2 \%, \mathrm{P}<0.001)$ and $5 \mathrm{Y}-\mathrm{OS}(97.0 \%$ vs. $83.2 \%, \mathrm{P}=0.002)$ than patients with elevated CEA ( $\geq 5 \mathrm{ng} / \mathrm{mL}$ ). There was no significant difference in 5Y-DFS and 5Y-OS according to the presence of tumor complications, tumor size, tumor differentiation, lymphovascular invasion,
Table 2. Multivariate analysis for 5-year DFS and 5-year OS in all patients

\begin{tabular}{llll}
\hline Variable & HR & \multicolumn{1}{c}{$95 \% \mathrm{Cl}$} & P-value \\
\hline 5-yr DFS & & & \\
$\quad$ Complication & 2.703 & $0.798-9.150$ & 0.110 \\
No. of retrieved LN & 5.009 & $1.827-13.733$ & 0.002 \\
Lymphovascular invasion & 0.300 & $0.037-2.464$ & 0.263 \\
Perineural invasion & 1.771 & $0.654-4.800$ & 0.261 \\
AC & 1.970 & $0.570-6.806$ & 0.284 \\
5-yr OS & & & \\
Complication & 4.050 & $1.166-14.061$ & 0.028 \\
No. of retrieved LN & 1.612 & $1.512-14.069$ & 0.007 \\
Perineural invasion & 1.891 & $0.631-5.664$ & 0.255 \\
AC & 1.456 & $0.408-5.193$ & 0.562 \\
\hline
\end{tabular}

DFS, disease-free survival; OS, overall survival; $\mathrm{HR}$, hazard ratio; $\mathrm{Cl}$, confidential interval; LN, lymph node; AC, adjuvant chemotherapy.

or perineural invasion.

The comparison of 5Y-DFS and 5Y-OS between the no-AC group and AC group is presented in Figs. 1 and 2, respectively. There was no significant difference in 5Y-DFS (94.8\% vs. $94.0 \%$, $\mathrm{P}=0.587)$ or $5 \mathrm{Y}-\mathrm{OS}(94.9 \%$ vs. $89.4 \%, \mathrm{P}=0.239)$ between the groups.

\section{DISCUSSION}

Age and performance score are considered to be the major factors that affect the decision to use AC for patients with stage IIA colon cancer because of the significant difference they exhibit between the no-AC and the AC groups. Jeon et al. [6] reported that surgical oncologists consider the patient's condition and postoperative state rather than high-risk factors to determine the use of AC. The 2 groups showed no significant difference in the presence of tumor complications, the number of retrieved LNs, lymphovascular invasion, or perineural invasion which are conventional risk factors for recurrence, and this may be because a combination of multiple factors was used to estimate the high-risk group.

The number of retrieved LNs was the only independent prognostic factor significantly affecting both $5 Y-D F S$ and $5 Y-O S$ by multivariate analysis. Although patients with less than 12 retrieved LNs had significantly poorer 5Y-DFS and 5Y-OS than the patients with 12 or more retrieved LNs in the no-AC group, both subgroups had similar survival outcomes in the AC group. Therefore, the number of retrieved LNs was considered to be the prognostic factor of stage IIA colon cancer, and patients with less than 12 retrieved LNs can benefit from AC. The number of retrieved LNs is one of the traditional risk factors for recurrence, and this is consistent with our findings. The number of retrieved LNs reflects the perfection of radical surgery and pathological evalua- 


\section{$\begin{array}{rlr}\text { Annals of } & \text { Prognostic Factor and } \\ \text { Coloproctology } & \text { Mok-Won Lee, et al. }\end{array}$}

Table 3. Correlation between clinicopathologic factors and survivals in the no adjuvant chemotherapy groups

\begin{tabular}{|c|c|c|c|c|c|}
\hline \multirow{2}{*}{ Variable } & \multirow{2}{*}{ No. of patient } & \multicolumn{2}{|c|}{ 5-yr DFS } & \multicolumn{2}{|c|}{5 -yr OS } \\
\hline & & Rate (\%) & P-value & Rate (\%) & P-value \\
\hline Sex & & & 0.557 & & 0.557 \\
\hline Male & 33 & 96.7 & & 96.6 & \\
\hline Female & 34 & 93.2 & & 93.1 & \\
\hline Age (yr) & & & 0.279 & & 0.251 \\
\hline$<75$ & 42 & 97.4 & & 97.3 & \\
\hline$\geq 75$ & 25 & 90.7 & & 90.5 & \\
\hline ASA PS classification & & & 0.625 & & 0.559 \\
\hline ।, || & 51 & 95.7 & & 95.7 & \\
\hline$I I I-V$ & 16 & 91.7 & & 91.7 & \\
\hline Sidedness & & & 0.279 & & 0.251 \\
\hline Right & 26 & 90.7 & & 90.5 & \\
\hline Left & 41 & 97.4 & & 97.3 & \\
\hline Tumor complication & & & 0.087 & & 0.133 \\
\hline No & 59 & 96.4 & & 96.2 & \\
\hline Yes & 8 & 80.0 & & 80.0 & \\
\hline CEA level (ng/mL) & & & 0.387 & & 0.381 \\
\hline$<5$ & 43 & 94.7 & & 94.6 & \\
\hline$\geq 5$ & 16 & 100 & & 100 & \\
\hline Tumor size (cm) & & & 0.088 & & 0.089 \\
\hline$<5$ & 35 & 90.2 & & 90.0 & \\
\hline$\geq 5$ & 32 & 100 & & 100 & \\
\hline No. of retrieved LN & & & 0.001 & & 0.001 \\
\hline$<12$ & 15 & 78.6 & & 78.6 & \\
\hline$\geq 12$ & 52 & 100 & & 100 & \\
\hline Differentiation & & & 0.687 & & 0.638 \\
\hline Well, moderate & 63 & 94.7 & & 94.5 & \\
\hline Poor, mucinous & 4 & 100 & & 100 & \\
\hline Lymphovascular invasion & & & 0.819 & & 0.817 \\
\hline No & 3 & 100 & & 100 & \\
\hline Yes & 64 & 94.9 & & 94.7 & \\
\hline Perineural invasion & & & 0.167 & & 0.170 \\
\hline No & 24 & 100 & & 100 & \\
\hline Yes & 43 & 91.8 & & 91.7 & \\
\hline
\end{tabular}

DFS, disease-free survival; OS, overall survival; ASA, American Society of Anesthesiologists; PS, physical status; CEA, carcinoembryonic antigen; LN, lymph node.

tion. Betge et al. [7] reported that the number of retrieved LNs was associated with higher tumor stage, tumor size, and rightsided location, and a low LN count indicates an adverse outcome in patients with locally advanced (T3/T4) disease. Therefore, efforts should be made to retrieve as many LNs as possible during surgery to complete radical surgery and aid pathological evaluation, even in the case of clinically N0 colon cancer, especially when it is suspected to be more than T3.

The presence of tumor complications was an independent prognostic factor significantly affecting $5 \mathrm{Y}-\mathrm{OS}$, according to multivariate analysis. Nevertheless, there was no significant difference in 5Y-DFS and 5Y-OS according to the presence of tumor complications between the groups. It is well-known that emergency surgery for colorectal cancer due to blood loss, obstruction, or perfo- 
Volume 37, Number 1, 2021

Ann Coloproctol 2021;37(1):35-43

Table 4. Correlation between clinicopathologic factors and survivals in the adjuvant chemotherapy groups

\begin{tabular}{|c|c|c|c|c|c|}
\hline \multirow{2}{*}{ Variable } & \multirow{2}{*}{ No. of patient } & \multicolumn{2}{|c|}{5 -yr DFS } & \multicolumn{2}{|c|}{5 -yr OS } \\
\hline & & Rate (\%) & P-value & Rate (\%) & P-value \\
\hline Sex & & & 0.538 & & 0.902 \\
\hline Male & 100 & 88.1 & & 94.5 & \\
\hline Female & 60 & 91.5 & & 93.2 & \\
\hline Age (yr) & & & 0.438 & & 0.117 \\
\hline$<75$ & 136 & 90.0 & & 95.4 & \\
\hline$\geq 75$ & 24 & 86.5 & & 83.7 & \\
\hline ASA PS classification & & & 0.639 & & 0.806 \\
\hline ।, || & 143 & 89.0 & & 94.1 & \\
\hline$\| I \mid-V$ & 17 & 93.8 & & 92.9 & \\
\hline Sidedness & & & 0.024 & & 0.019 \\
\hline Right & 51 & 97.8 & & 100 & \\
\hline Left & 109 & 85.6 & & 91.2 & \\
\hline Tumor complication & & & 0.661 & & 0.268 \\
\hline No & 104 & 90.0 & & 92.9 & \\
\hline Yes & 39 & 85.9 & & $100^{\mathrm{a}}$ & \\
\hline CEA level (ng/mL) & & & $<0.001$ & & 0.002 \\
\hline$<5$ & 137 & 94.9 & & 97.9 & \\
\hline$\geq 5$ & 23 & 72.2 & & 83.2 & \\
\hline Tumor size (cm) & & & 0.229 & & 0.201 \\
\hline$<5$ & 77 & 86.2 & & 92.9 & \\
\hline$\geq 5$ & 83 & 92.3 & & 94.9 & \\
\hline No. of retrieved LN & & & 0.098 & & 0.316 \\
\hline$<12$ & 34 & 80.8 & & 90.2 & \\
\hline$\geq 12$ & 126 & 91.7 & & 94.9 & \\
\hline Differentiation & & & 0.559 & & 0.560 \\
\hline Well, moderate & 156 & 89.2 & & 93.8 & \\
\hline Poor, mucinous & 4 & 100 & & 100 & \\
\hline Lymphovascular invasion & & & 0.172 & & 0.625 \\
\hline No & 3 & 66.7 & & 100 & \\
\hline Yes & 157 & 89.8 & & 93.8 & \\
\hline Perineural invasion & & & 0.838 & & 0.954 \\
\hline No & 64 & 89.8 & & 94.9 & \\
\hline Yes & 96 & 89.1 & & 93.3 & \\
\hline
\end{tabular}

DFS, disease-free survival; OS, overall survival; ASA, American Society of Anesthesiologists; PS, physical status; CEA, carcinoembryonic antigen, LN, lymph node.

a-year OS, 59.3\%.

ration is associated not only with high postoperative mortality but also with poor cancer-specific survival. The reason for this is multifactorial. Systemic inflammatory response may release proinflammatory cytokines and growth factors that promote tumor growth and compromise immune function [8]. This leads to the elevation of C-reactive protein (CRP), which has been reported to be associated with poor survival [9]. Okuda et al. [10] reported that obstruction was an independent poor prognostic factor for stage II colorectal cancer and that even stage II colorectal cancers with obstruction showed poorer prognosis than stage III colorectal cancers without obstruction. They suggested the ability of the tumor to expand inside resulting in colon obstruction is as signifi- 


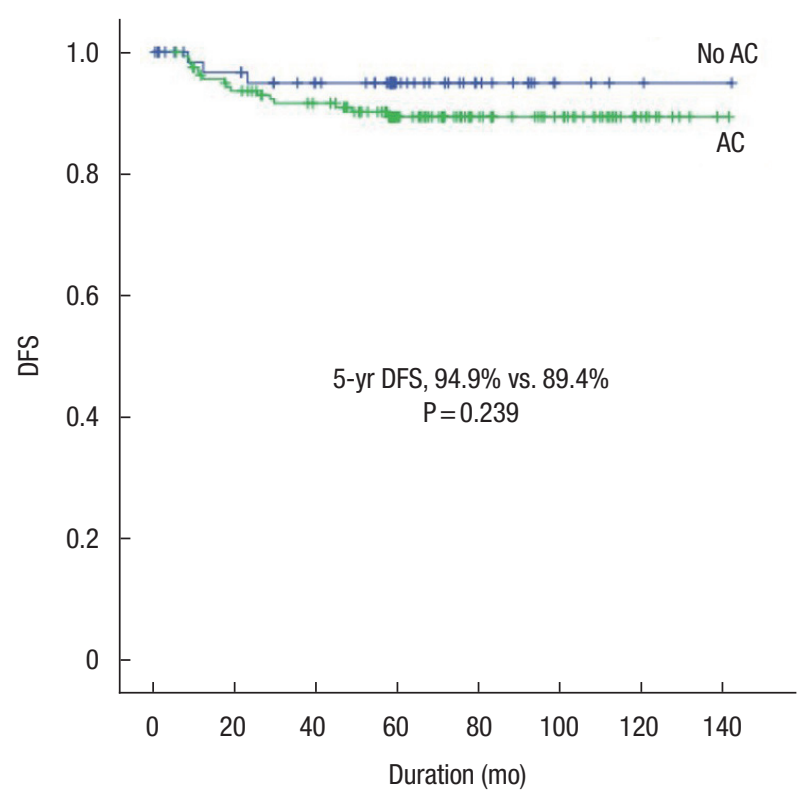

Fig. 1. Comparisons of 5-year disease-free survival (DFS) between adjuvant chemotherapy (AC) group and no-AC group.

cant for poor survival as its ability to expand outside, that is, pT4. Based on the definition of stage IIA colon cancer, it cannot have pT4; however, clinical situations such as obstruction or perforation can indicate that these cancers belong to an advanced subgroup of $\mathrm{pT} 3$, and therefore, $\mathrm{T}$ staging becomes significant. This suggests that a different $\mathrm{T}$ staging system that is more specific and accurate than the current system is needed.

Although there was no significant difference in the 5Y-DFS and $5 Y-O S$ between the patients with left and right colon cancer in the no-AC group, the patients with right colon cancer had a significantly better 5Y-DFS and 5Y-OS than those with left colon cancer in the AC group. Although sidedness is not a prognostic factor for survival, it can be a predictive factor for response to AC in stage IIA colon cancer. Based on the present result, it may be presumed that patients with right colon cancer could benefit from AC while those with left colon cancer would not.

Many researches have reported the differences between left and right colon cancers at the various aspects of epidemiology, clinical course, survival, genetics, molecular biology, and microbiology. Generally, right colon cancer has been shown to be associated with poor differentiation, advanced-stage disease, distinct genetic mutations, and inferior prognosis, especially in stage III and IV colon cancers [11]. Weiss et al. [12] reported the mortality by stage for right and left colon cancers. They reported that there was no overall difference in the 5-year mortality rate between right and left colon cancers. However, for stage II disease, right colon cancer had lower mortality, while for stage III, right colon cancers had higher mortality than left colon cancer [12]. The differences by sidedness were considered due to complex mechanisms of ge-

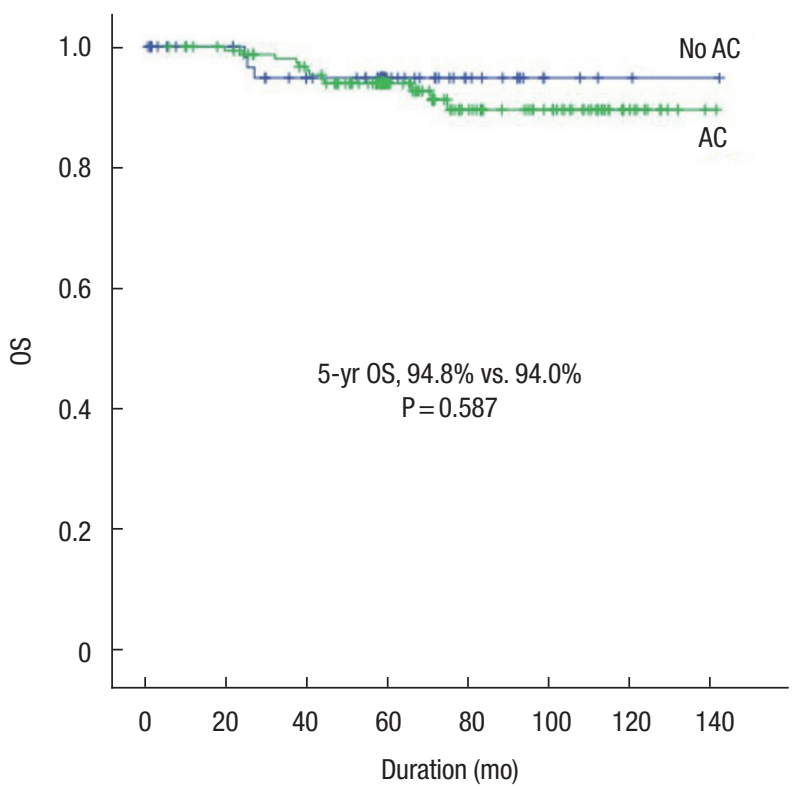

Fig. 2. Comparisons of 5-year overall survival (OS) between adjuvant chemotherapy (AC) group and no-AC group.

netic and epigenetic changes caused by inherited and environmental factors and insisted that not only the tumor stage or grade but also molecular mechanisms of carcinogenesis related to the anatomical site needs to be considered for the management of colon cancer. Guinney et al. [13] presented the international consortium for 4 consensus molecular subtypes (CMS) of colorectal cancers. The characteristics of CMS1 tumors, also known as microsatellite instability immune type, are microsatellite instability, CpG island methylator phenotype-high, BRAF mutations, and immune infiltration and activation, and these tumors are frequently diagnosed in right colon cancers. Notably, the CMS1 population had a very poor survival rate after relapse, in agreement with many studies reporting poor prognosis for patients with BRAF mutations. A systematic review and meta-analysis by Petrelli et al. [14] reported that left colon cancer was associated with a significantly lower mortality, and this was independent of stage, race, or AC. Based on these results, patients with right colon cancers might have a chance to improve survival with AC because of their poor prognosis, and the results of the present study support this possibility. Petrelli et al. [14] insisted that sidedness should be acknowledged as a criterion for establishing prognosis in all stages of disease, and it should be considered when deciding treatment in metastatic setting. Moreover, it represents a stratification factor for future adjuvant treatment. For metastatic colon cancer, sidedness should be considered for the selection of targeted therapy. However, its significance for AC in nonmetastatic colon cancer was barely studied until now. In this era of precision medicine, we need further studies about stratification factors like sidedness, especially for controversial population with stage IIA 
colon cancer.

Although there was no significant difference in the 5Y-DFS and 5Y-OS between patients with normal and elevated CEA in the noAC group, the patients with normal CEA had a significantly better 5Y-DFS and 5Y-OS than those with elevated CEA in the AC group. Although it is difficult to presume that benefit of AC would be correlated with CEA based on the result of the present study, CEA may be one of the potential predictive factors for efficacy of AC.

CEA is a traditional tumor marker for monitoring the recurrence of several tumors including colon cancer, and elevated preoperative CEA has been considered to be related to a poor prognosis [15]. Many studies have reported that CEA was a reliable predictor of recurrence and poor survival particularly in stage II colon cancer and they supported the use of CEA to guide AC in stage II colon cancer [16]. Although many of them suggested that AC should be considered for patients with elevated CEA [17], a large population-based and propensity score matching study by Liu et al. [18] reported that AC did not show substantial survival benefit in stage IIA colon cancer with elevated pretreatment CEA, and they insisted that stage IIA colon cancer with elevated pretreatment CEA should not be treated with AC. Recently, several studies have reported the significance of postoperative CEA for the prediction of recurrence and survival, not only preoperative CEA. A post hoc analysis of the MOSAIC trial reported that use of oxaliplatin showed survival benefit only in patients with high-risk stage II colon cancer with more than $2.35 \mathrm{ng} / \mathrm{mL}$ of postoperative CEA and suggested the addition of oxaliplatin to 5-FU only for those patients [17].

In the present study, there was no significant difference in the 5Y-DFS and 5Y-OS of differentiation, lymphovascular invasion, and perineural invasion which are traditional risk factors defined by current worldwide guidelines. The significance of these risk factors to decide the use $\mathrm{AC}$ for stage IIA colon cancer needs to be reevaluated. A population-based cohort study by Osterman et al. [19] investigated 'emerging' risk factors (sidedness, pT3 and pT4 subclassification, LN ratio, tumor deposits, and pre- and postoperative CEA and CRP level) which were distinguished from 'classic' risk factors (emergency surgery, $\mathrm{pT}$ and $\mathrm{pN}$ classification, low LN yield, malignancy grade, vascular and perineural invasion, and adjuvant treatment) using traditional guidelines. They reported that $\mathrm{pN}$ subclassification, sidedness, and elevated postoperative CEA correlated with recurrence after adjusting for $\mathrm{T}$ and $\mathrm{N}$ stage and high-risk factors by NCCN guideline. The results of the present study also show a low significance of classic prognostic factors including differentiation, lymphovascular invasion, and perineural invasion, and possible significance of other predictable factors for benefit by AC including sidedness and CEA.

There was no significant difference in survival outcomes between the no- $\mathrm{AC}$ group and the $\mathrm{AC}$ group. The present results suggest that there was no benefit of AC for patients with stage IIA colon cancer. There are many studies that are not supportive of
AC for resected stage II colon cancer, including those comparing 5-FU to observation [20-23] and FOLFOX to 5-FU without oxaliplatin [24, 25]. O'Connor et al. [26] reported that AC did not substantially improve OS in stage II colon cancer, with or without poor prognostic factors. Because stage IIB, in which the tumor penetrates to the visceral peritoneum, and IIC, in which it invades or is adherent to other organs or structures, are considered as definite risk factors for recurrence $[27,28], \mathrm{AC}$ is considered to be beneficial for them [29]. However, there was a relative lack of research for stage IIA colon cancer. Pinto et al. [30] reported on the overall good prognosis and absence of recurrence predictive factors of stage IIA colon cancer and validated the decision of not submitting the patients to chemotherapy risks. However, although stage IIA colon cancer has a relatively good prognosis, $20 \%$ to $25 \%$ of patients eventually die of recurrence. This suggests that AC could still be essential for some patients with stage IIA colon cancer. However, because the development of examination for preoperative staging has decreased the rate of false stage II colon cancer (which are actually stage IV disease) diagnosis and the progress of surgical techniques such as complete mesocolic excision has enabled more precise pathologic diagnosis and improved prognosis than before, the proportion of patients with stage IIA colon cancer who would benefit from AC is considered to have decreased. Therefore, it is necessary to refine and reinforce indications for AC. We need further studies on not only the aforementioned classic and emerging factors but also the microsatellite instability, genetic mutations, microRNA, circulating tumor DNA, or tumor microenvironment to predict prognosis more precisely than before and to select the patients who can acquire survival benefit. Consequently, personalized and precision medicine should be introduced not only for metastatic colon cancer but also for nonmetastatic colon cancer with respect to $\mathrm{AC}$ and surveillance.

The main limitations of this study are the small cohort size from single institution and the retrospective study design. Heterogeneity of chemotherapeutic regimens and inconsistent indication for $\mathrm{AC}$ can also be major bias. Therefore, we need more an optimally designed prospective study with a large cohort.

In conclusion, the number of retrieved LNs and the presence of tumor complications were prognostic factors for stage IIA colon cancer, but not lymphovascular and perineural invasion. Sidedness and preoperative CEA could be used as predictive factors to decide on the benefit of AC. According to our study, it is considered that there was no benefit of AC for stage IIA colon cancer. Further study for the selection of the patients who can acquire survival benefit of $\mathrm{AC}$ is needed.

\section{CONFLICT OF INTEREST}

No conflicts of interest relevant to this study were reported. 


\section{REFERENCES}

1. National Cancer Information Center. Incidence of cancer by site, 2017 [Internet]. Goyang (KR): National Cancer Information Center; 2019 [cited 2020 May 16]. Available from: https://cancer. go.kr/lay1/S1T639C641/contents.do.

2. National Cancer Information Center. Five-year relative survival rate of cancer by site and summary stage, 2013-2017. Goyang (KR): National Cancer Information Center; 2019 [cited 2020 May 16]. Available from: https://cancer.go.kr/lay1/S1T648C652/contents.do.

3. Shi Q, Sobrero AF, Shields AF, Yoshino T, Paul J, Taieb J, et al. Prospective pooled analysis of six phase III trials investigating duration of adjuvant (adjuv) oxaliplatin-based therapy (3 vs 6 months) for patients (pts) with stage III colon cancer (CC): The IDEA (International Duration Evaluation of Adjuvant chemotherapy) collaboration. J Clin Oncol 2017;35(18 Suppl):LBA1.

4. Iveson T, Sobrero AF, Yoshino T, Sougklakos I, Ou FS, Meyers JP, et al. Prospective pooled analysis of four randomized trials investigating duration of adjuvant (adj) oxaliplatin-based therapy (3 vs 6 months $\{\mathrm{m}\}$ ) for patients (pts) with high-risk stage II colorectal cancer (CC). J Clin Oncol 2019;37(15 Suppl):3501.

5. West NP, Hohenberger W, Weber K, Perrakis A, Finan PJ, Quirke P. Complete mesocolic excision with central vascular ligation produces an oncologically superior specimen compared with standard surgery for carcinoma of the colon. J Clin Oncol 2010; 28:272-8.

6. Jeon $\mathrm{CH}$, Kim MK, Lee IK. Indication for and effect of adjuvant chemotherapy for stage IIa (T3N0M0) colon cancer. Ann Coloproctol 2019;35:254-61.

7. Betge J, Harbaum L, Pollheimer MJ, Lindtner RA, Kornprat P, Ebert MP, et al. Lymph node retrieval in colorectal cancer: determining factors and prognostic significance. Int J Colorectal Dis 2017;32:991-8.

8. Coussens LM, Werb Z. Inflammation and cancer. Nature 2002; 420:860-7.

9. Allin KH, Bojesen SE, Nordestgaard BG. Baseline C-reactive protein is associated with incident cancer and survival in patients with cancer. J Clin Oncol 2009;27:2217-24.

10. Okuda Y, Shimura T, Yamada T, Hirata Y, Yamaguchi R, Sakamoto $\mathrm{E}$, et al. Colorectal obstruction is a potential prognostic factor for stage II colorectal cancer. Int J Clin Oncol 2018;23:1101-11.

11. Schrag D, Weng S, Brooks G, Meyerhardt JA, Venook AP. The relationship between primary tumor sidedness and prognosis in colorectal cancer. J Clin Oncol 2016;34(15 Suppl):3505.

12. Weiss JM, Pfau PR, O'Connor ES, King J, LoConte N, Kennedy G, et al. Mortality by stage for right- versus left-sided colon cancer: analysis of surveillance, epidemiology, and end results. Medicare data. J Clin Oncol 2011;29:4401-9.

13. Guinney J, Dienstmann R, Wang X, de Reyniès A, Schlicker A, Soneson C, et al. The consensus molecular subtypes of colorectal cancer. Nat Med 2015;21:1350-6.
14. Petrelli F, Tomasello G, Borgonovo K, Ghidini M, Turati L, Dallera P, et al. Prognostic survival associated with left-sided vs right-sided colon cancer: a systematic review and meta-analysis. JAMA Oncol 2017;3:211-9.

15. Wanebo HJ, Rao B, Pinsky CM, Hoffman RG, Stearns M, Schwartz MK, et al. Preoperative carcinoembryonic antigen level as a prognostic indicator in colorectal cancer. N Engl J Med 1978; 299:448-51.

16. Peng Y, Wang L, Gu J. Elevated preoperative carcinoembryonic antigen (CEA) and Ki67 is predictor of decreased survival in IIA stage colon cancer. World J Surg 2013;37:208-13.

17. Auclin E, André T, Taieb J, Banzi M, Van Laethem JL, Tabernero J, et al. Association of post-operative CEA with survival and oxaliplatin benefit in patients with stage II colon cancer: a post hoc analysis of the MOSAIC trial. Br J Cancer 2019;121:312-7.

18. Liu Q, Huang Y, Luo D, Zhang S, Cai S, Li Q, et al. Evaluating the guiding role of elevated pretreatment serum carcinoembryonic antigen levels for adjuvant chemotherapy in stage IIA colon cancer: a large population-based and propensity score-matched study. Front Oncol 2019;9:37.

19. Osterman E, Mezheyeuski A, Sjöblom T, Glimelius B. Beyond the NCCN risk factors in colon cancer: an evaluation in a Swedish population-based cohort. Ann Surg Oncol 2020;27:1036-45.

20. International Multicentre Pooled Analysis of B2 Colon Cancer Trials (IMPACT B2) Investigators. Efficacy of adjuvant fluorouracil and folinic acid in B2 colon cancer. J Clin Oncol 1999;17:135663.

21. Gill S, Loprinzi CL, Sargent DJ, Thomé SD, Alberts SR, Haller DG, et al. Pooled analysis of fluorouracil-based adjuvant therapy for stage II and III colon cancer: who benefits and by how much? J Clin Oncol 2004;22:1797-806.

22. Figueredo A, Charette ML, Maroun J, Brouwers MC, Zuraw L. Adjuvant therapy for stage II colon cancer: a systematic review from the Cancer Care Ontario Program in evidence-based care's gastrointestinal cancer disease site group. J Clin Oncol 2004;22: 3395-407.

23. Quasar Collaborative Group; Gray R, Barnwell J, McConkey C, Hills RK, Williams NS, et al. Adjuvant chemotherapy versus observation in patients with colorectal cancer: a randomised study. Lancet 2007;370:2020-9.

24. André T, Boni C, Navarro M, Tabernero J, Hickish T, Topham C, et al. Improved overall survival with oxaliplatin, fluorouracil, and leucovorin as adjuvant treatment in stage II or III colon cancer in the MOSAIC trial. J Clin Oncol 2009;27:3109-16.

25. Kuebler JP, Wieand HS, O'Connell MJ, Smith RE, Colangelo LH, Yothers G, et al. Oxaliplatin combined with weekly bolus fluorouracil and leucovorin as surgical adjuvant chemotherapy for stage II and III colon cancer: results from NSABP C-07. J Clin Oncol 2007;25:2198-204.

26. O'Connor ES, Greenblatt DY, LoConte NK, Gangnon RE, Liou JI, Heise CP, et al. Adjuvant chemotherapy for stage II colon cancer with poor prognostic features. J Clin Oncol 2011;29:3381-8. 
27. Quah HM, Chou JF, Gonen M, Shia J, Schrag D, Landmann RG, et al. Identification of patients with high-risk stage II colon cancer for adjuvant therapy. Dis Colon Rectum 2008;51:503-7.

28. O'Connell JB, Maggard MA, Ko CY. Colon cancer survival rates with the new American Joint Committee on Cancer sixth edition staging. J Natl Cancer Inst 2004;96:1420-5.

29. Sargent D, Sobrero A, Grothey A, O'Connell MJ, Buyse M, Andre
T, et al. Evidence for cure by adjuvant therapy in colon cancer: observations based on individual patient data from 20,898 patients on 18 randomized trials. J Clin Oncol 2009;27:872-7.

30. Pinto JC, Rosa I, Martins C, Marques I, da Silva JP, Fonseca R, et al. Colon adenocarcinoma stage IIA: can we predict relapse? J Gastrointest Cancer 2020;51:116-20. 